\title{
Die „Neue Dolomitenbahn“6. Das Herzstück der Vision einer alpinen Eisenbahnlinie von Genf nach Venedig.
}

\author{
Christoph Perathoner und Helmuth Moroder
}

\section{Der verkehrspolitische Rahmen}

Mit der Eröffnung des Brenner-Basistunnels (BBT) ${ }^{1}$ und der Fertigstellung wichtiger Eisenbahnverbindungen vor allem in Deutschland, Österreich und Frankreich aber auch in Italien - wird Bozen, als Landeshauptstadt der Autonomen Provinz Bozen-Südtirol, sicherlich in der Nord-Süd-Verbindung ganz neue verkehrstechnische Potenziale ausschöpfen können. Von Bozen aus werden Dank des BBT innerhalb weniger Stunden wichtige Städte und Handelszentren wie München, Berlin, Paris, Prag oder Frankfurt erreichbar sein. Diesen Meilenstein der Mobilität macht

\footnotetext{
${ }^{1}$ Ital. Galleria di Base del Brennero; Beim BBT handelt es sich um ein Gemeinschaftsprojekt der EU-Mitgliedstaaten Österreich und Italien im Rahmen der TEN-V-Politik der Europäischen Union. Das Vorhaben, das nach heutigem Stand erst im Jahr 2032 beendet werden soll, sieht den Bau eines Eisenbahntunnels für den Personen- wie auch Güterverkehr unter dem Brennerpass zwischen Innsbruck (AUT) und Franzensfeste/Fortezza (ITA) mit einer Länge von rund $55 \mathrm{~km}$ (mit der Umfahrung von Innsbruck 65 km) vor; vgl. Konrad Bergmeister, Brenner Basistunnel - Brenner Base Tunnel - Galleria di Base del Brennero, Tappeiner, Bozen/Innsbruck, 2011; Jutta Kusstatscher (Hrsg.), Tunnelblick. Der Brennerbasistunnel. Fakten - Argumente - Meinungen, Studien Verlag, Innsbruck 2007 in: https://www.bbt-se.com (30.05.2020).
}

Article note: Helmuth Moroder ist Leiter der Abteilung Busdienst der SAD Nahverkehr AG. Der Verfasser bringt in diesem Beitrag allein seine persönliche Auffassung zum Ausdruck.

\author{
C. Perathoner $(\bowtie)$ \\ Rechtsanwaltssozietät Christoph Perathoner \& Partner, Bozen, Italien \\ E-Mail: christoph.perathoner@ perathoner-partner.com \\ H. Moroder \\ SAD Nahverkehr AG, Bozen, Italien \\ E-Mail: helmuth.moroder@sad.it
}

S. Laimer, C. Perathoner (Hrsg.), Mobilitäts- und Transportrecht in Europa,

Bibliothek des Wirtschaftsrechts 2,

https://doi.org/10.1007/978-3-662-63635-0_13 
die Politik des Transeuropäischen Verkehrsnetzes (TEN-V) ${ }^{2}$ der Europäischen Union möglich.

Die Europäische Union strebt die Entwicklung eines europaweiten Netzes an Eisenbahnlinien, Straßen, Binnenwasserstraßen, Seeschifffahrtswegen, Häfen, Flughäfen und Eisenbahnterminals an. Das Ziel dieses groß angelegten Verkehrskonzeptes für den europäischen Kontinent besteht darin, Verkehrslücken zu schließen, Engpässe und technische Barrieren zu beseitigen sowie den sozialen, wirtschaftlichen und territorialen Zusammenhalt in der EU zu stärken und somit auch die Grundlage für die Entwicklung des Binnenmarktes und das Zusammenwachsen der Menschen zu erwirken. ${ }^{3}$

Die Europäische Union hat allerdings in der eigenen TEN-V-Politik keine von Westen nach Osten durch die Alpen querende Verkehrsverbindung vorgesehen, was aus Sicht der EU durchaus verständlich und nachvollziehbar ist. Dabei wäre eine derartige Verbindung im Alpenraum nicht nur eng mit Nachhaltigkeit, Natur- und Landschaftsschutz und der Wahrung der Schönheit dieses Gebietes verbunden, sondern würde sich insbesondere auch positiv auf den öffentlichen Nahverkehr sowie auf den Tourismussektor auswirken.

Einige Avantgarde-Projekte in den Alpen beweisen, dass die Bahn seit Jahrzehnten auch in Bergregionen ein ideales Verkehrsmittel darstellen kann. Beispielhaft bezeugt dies der Glacier-Express. ${ }^{4}$ Der „langsamste Schnellzug der Welt" ${ }^{\star 5}$ bringt Fahrgäste seit 1930 über 291 Brücken, durch 91 Tunnel und über den 2033 m hohen Oberalppass nahe der Quelle des Rheins. Heute gilt der Glacier-Express als eine der bedeutendsten touristischen Attraktionen in Europa ${ }^{6}$ Ein nicht weniger eindrucksvolles Bahnprojekt ist der Bernina-Express (BEX), ${ }^{7}$ der zwischen St. Moritz $(\mathrm{CH})$ und Tirano (ITA) verkehrt.

\footnotetext{
${ }^{2}$ Engl. Trans-European Transport Network (TEN-T); vgl. online: https://ec.europa.eu/transport/ themes/infrastructure/ten-t_en (zugegriffen am 30.05.2020).

${ }^{3}$ Die wichtigste Rechtsgrundlage der TEN-T Politik der EU bildet bislang die Verordnung (EU) Nr. 1315/2013 des Europäischen Parlaments und des Rates vom 11. Dezember 2013 über Leitlinien der Union für den Aufbau eines transeuropäischen Verkehrsnetzes und zur Aufhebung des Beschlusses Nr. 661/2010/EU, in: ABl. vom 20.12.2013, L 348, S. 1-128; ELI: http://data.europa. eu/eli/reg/2013/1315/oj (zugegriffen am 30.05.2020).

${ }^{4}$ Vgl. https://www.glacierexpress.ch/de (zugegriffen am 30.05.2020).

${ }^{5}$ Vgl. Ronald Gohl, Der Glacier Express. Rund um den langsamsten Schnellzug der Welt, Geramond, München 2000; Hans Schweers, Glacier Express. Der langsamste Schnellzug der Welt, Schweers und Wall, Aachen, 1991.

${ }^{6}$ Vgl. Reto Steiner (Hrsg.), Glacier Express - Von St. Moritz nach Zermatt, EK-Verlag, Freiburg, 2009; Robert Bösch/Iso Camartin/Paul Caminada, Glacier Express. Die Welt des Glacier Express, AS Verlag, Zürich, 2008.

${ }^{7}$ Robert Bösch/Iso Camartin/Gion Caprez, Bernina Express, AS Verlag, Zürich, 2009 in: https:// www.rhb.ch/de/panoramazuege/bernina-express (zugegriffen am 30.05.2020).
} 


\section{Die Vision einer Eisenbahnlinie zwischen Genf und Venedig}

Vor wenigen Jahren präsentierte das Südtiroler Unternehmen SAD Nahverkehr AG/ SAD Trasporto locale S.p.A., ${ }^{8}$ welches im öffentlichen Personennahverkehr tätig ist, eine verkehrstechnische und verkehrspolitische Vision einer von Osten nach Westen die Alpen überquerenden Eisenbahnverbindung.

Der Grundgedanken besteht darin, zur Vervollständigung eines alpinen Verkehrskreuzes, nach der Verwirklichung der Eisenbahnachse Berlin - Palermo, dem TEN-Prioritätsprojekt Nr. 1, das von Norden nach Süden verläuft, auch eine alpine Eisenbahnachse von Westen nach Osten zu schaffen. Diese sollte in Genf/Genève $(\mathrm{CH})$ beginnen und in Venedig/Venezia (ITA) enden. Von Genf aus sind Lyon und Marseille und somit das französische und spanische Eisenbahnnetz leicht erreichbar. Von Venedig aus kann über Triest der Osten Europas eisenbahnmäßig erschlossen werden.

Diese Verbindung würde sich in ihrer Funktion jedoch tief greifend von jener der TEN-V Achsen unterscheiden: Sie sollte hauptsächlich dem Personenverkehr dienen und nicht eine Schnellstrecke darstellen, sondern für den regionalen Nahverkehr konzipiert sein. Der Zusammenschluss von Genf und Venedig würde die touristische Attraktivität der Verbindung maßgeblich steigern, aber bereits der Ausbau von regionalen Teilstücken, wie z. B. die Dolomitenbahn von Bozen bis Cortina d'Ampezzo, wäre für die betreffende Region äußerst bedeutsam.

\footnotetext{
${ }^{8}$ Die Präsentation der neuen Bahnvision stieß auf ein sehr starkes Medienecho und die öffentliche Diskussion zog sich in Südtirol über viele Monate. Der damalige italienische Vizeminister für Infrastruktur und Verkehr Riccardo Nencini richtete für dieses Projekt einen Arbeitstisch im italienischen Transportministerium ein. Am 27. September 2016 fand schließlich unter dessen Vorsitz das Treffen in Rom statt, bei dem neben der SAD-Delegation auch der damalige Generaldirektor des Transportministeriums Virginio Di Giambattista sowie Führungskräfte vom Gruppo Ferrovie dello Stato Italiane anwesend waren. Mit dem Fall der Regierung Matteo Renzi im Dezember 2016 wurde der Arbeitstisch aufgelöst und bis heute nicht wiedereingerichtet. Zur Vorstellung des Projektes siehe: Stefan Pfeifhofer, Die 1,6 Milliarden-Euro-Bahn, in: Dolomiten. Tagblatt der Südtiroler, 30./31. Juli 2016, S. 14; Thomas Vikoler, Die schönste Bahn der Welt, in: Neue Südtiroler Tageszeitung, 30./31. Juli 2016, S. 9; Federico Sanzovo, Un treno per salvare i monti dalle auto. Presentato il progetto del collegamento tra Bolzano e Cortina che potrebbe essere usato da 7,5 milioni di turisti all'anno, in: Alto Adige - Quotidiano indipendente fondato nel 1945, 30. Juli 2016, S. 24; zur öffentlichen Diskussion im Anschluss an die Präsentation siehe: Hatto Schmidt, Die Grödner meinen: Eine tolle Sache. Dolomitenbahn: Projekt der SAD für eine Bahnlinie von Bozen nach Anpezo/Cortina d'Ampezzo vorgestellt, in: Dolomiten. Tagblatt der Südtiroler, 14. Oktober 2016, S. 19; Die Gadertaler sind für die Bahn, in: Dolomiten. Tagblatt der Südtiroler, 27. Oktober 2016; Benno Zöggeler, Die Dolomitenbahn wird kommen, in: Dolomiten. Tagblatt der Südtiroler, 10./11. Dezember 2016, S. 27; zum Arbeitstisch im italienischen Transportministerium siehe: Heinrich Schwarz, Die Dolomitenbahn in Rom. Vizeminister Riccardo Nencini leitete am Dienstag ein Treffen zum 1,6-Milliarden-Projekt Dolomitenbahn. Die Details, in: Neue Südtiroler Tageszeitung, 29. September 2016; Dolomitenbahn nimmt Fahrt auf, in: Dolomiten. Tagblatt der Südtiroler, 29. September 2016; David Lardschneider, La Ferata dla Dolomintes cun stazion a Roma, in: La Usc di Ladins, 7. Oktober 2016, S. 4-5.
} 


\subsection{Die Trasse}

Innerhalb der Schweiz besteht bereits ein ausreichendes Eisenbahnnetz von Genf bis nach Scuol. Die Trasse führt dabei über eine Normalspur (standard gauge) auf dem Netz der Schweizerischen Bundesbahnen AG (SBB $)^{9}$ von Genf über Lausanne, Fribourg, Bern, Zürich bis nach Landquart.

$\mathrm{Ab}$ Landquart führt die Trasse über Klosters Platz zum Bahnhof Scuol-Tarasp in Graubünden auf einer Schmalspur (narrow gauge) des schweizerischen Eisenbahnverkehrs- und Eisenbahninfrastrukturunternehmens Rhätische Bahn (RhB). ${ }^{10}$

Vom Bahnhof Scuol-Tarasp (CH) bis nach Mals/Malles Venosta (ITA) fehlt es an einer Bahnverbindung. Eine solche müsste errichtet werden, wobei es sich um keine unmögliche Aufgabe handeln dürfte, zumal Mals im Vinschgau rund 22 km Luftlinie von Scuol entfernt ist.

Ab Mals fährt die Vinschgaubahn, ${ }^{11}$ die von der SAD Nahverkehr AG als Eisenbahnverkehrsunternehmen betrieben wird, auf einer Normalspurbahn der Südtiroler Transportstrukturen AG (STA), einer In-house-Gesellschaft der Autonomen Provinz Bozen-Südtirol, bis Meran/Merano (ITA).

Von Meran führt dann die Meraner Bahnlinie, ${ }^{12}$ eine einspurige Normalbahn unter der Verwaltung von Rete Ferroviaria Italiana (RFI), ${ }^{13}$ nach Bozen.

\footnotetext{
${ }^{9}$ Franz. Chemins de fer fédéraux suisses (CFF), ital. Ferrovie federali svizzere (FFS), rätoroman. Viafiers federalas svizras (VFF), engl. Swiss federal railways (SFR); vgl. Heinz von Arx (Hrsg.), Der Kluge reist im Zuge - Hundert Jahre SBB, AS-Verlag, Zürich, 2001; Ronald Gohl, Die Schweizer Bundesbahnen. Geschichte - Strecken - Fahrzeuge, 2. Aufl., GeraMond, München 2009, in: https://www.sbb.ch (zugegriffen am 30.05.2020).

${ }^{10}$ Ital. Ferrovia retica, rätoroman. Viafier retica. Die RhB betreibt das größte zusammenhängende Schmalspur-Netz der Schweiz; vgl. Hans-Bernhard Schönborn, Die Rhätische Bahn. Geschichte und Gegenwart, GeraMond, München, 2009, in: https://www.rhb.ch (zugegriffen am 30.05.2020).

${ }^{11}$ Auch Vinschger Bahn, ital. Ferrovia della Val Venosta. Die Vinschgaubahn wurde 1906 eröffnet und nach dem Ersten Weltkrieg von den Italienischen Staatsbahnen/Ferrovie dello Stato übernommen. Die Bahn wurde im Jahre 1990 aufgelassen. Auf Betreiben des Landes Südtirol wurde die Bahn im Jahre 2005 wiedereröffnet; vgl. Peter Hilpold, Die Reaktivierung der Vinschgerbahn. Eine verkehrsgeografische Analyse der 2005 wieder in Betrieb genommenen Nebenbahn Meran Mals (Italien/Südtirol), VDM Verlag Dr. Müller, Saarbrücken, 2008; Andreas Gottlieb Hempel, Vinschgau in einem Zug - mit der Bahn durch das westliche Südtirol - wandern, Rad fahren, Kultur erleben, Folio-Verlag, Wien/Bozen 2006, in: https://www.sta.bz.it/de/bahnhoefe-zuege/ vinschger-bahn (zugegriffen am 30.05.2020).

${ }^{12}$ Auch Bahnstrecke Bozen - Meran; ital. Ferrovia Bolzano - Merano; Vgl. https://www.sta.bz.it/ de/bahnhoefe-zuege/die-meraner-bahnlinie (zugegriffen am 30.05.2020).

${ }^{13} \mathrm{Vgl}$. http://www.rfi.it (zugegriffen am 30.05.2020). RFI ist eine Tochtergesellschaft der Ferrovie dello Stato Italiane (FS; deut. Italienische Staatseisenbahnen) und ist innerhalb der Unternehmensfamilie der Staatsbahnen für den Bereich des Schienennetzes und Eisenbahninfrastruktur zuständig. Die Tochtergesellschaft Trenitalia ist hingegen für den Personen- und Güterverkehr zuständig; vgl. Massimo Centra (Hrsg.), Ferrovia e società. Il centenario delle Ferrovie dello Stato, Il Mulino, Bologna, 2006, in: https://www.fsitaliane.it (zugegriffen am 30.05.2020).
} 
In Bozen (Region Trentino-Südtirol, Provinz Bozen-Südtirol) sollte dann die neue Dolomitenbahn starten, die zuerst für einen kurzen Teil der Brennerbahn ${ }^{14}$ folgen und dann über das Schlerngebiet und Gröden nach Cortina d'Ampezzo (Region Venetien/Veneto, Provinz Belluno) führen würde.

Von Cortina d'Ampezzo aus müsste wiederum eine Eisenbahnlinie errichtet werden, um den nächstgelegenen Bahnhof zu erreichen, der sich in Calalzo di Cadore (Venetien/Veneto, Provinz Belluno), rund $30 \mathrm{~km}$ weiter südlich befindet. Eine Eisenbahnverbindung zwischen Cortina und Calalzo bestand bereits im Rahmen der alten Dolomitenbahn bis zum Mai 1964. ${ }^{15}$ Ab Calalzo besteht bereits eine Eisenbahnlinie, welche über Belluno nach Venedig/Venezia Santa Lucia führt. Netzbetreiber letztgenannter Bahnlinie ist RFI, während der Dienst von Trenitalia abgewickelt wird.

\section{Die ,Neue Dolomitenbahn“6}

\subsection{Der sozioökonomische Rahmen}

Das Land Südtirol ist eine im Herzen der Alpen eingebettete Tourismusregion, welche sowohl im Sommer als auch im Winter auf zwei starke Saisonen aufbaut. Laut dem Südtiroler Landesinstitut für Statistik ASTAT ${ }^{16}$ verzeichneten die Beherbergungsbetriebe im Tourismusjahr 2018/2019 rund 33,6 Millionen Nächtigungen und 7,7 Millionen Ankünfte. ${ }^{17}$ Das Grödental ${ }^{18}$ wie auch das Hochabteital ${ }^{19}$ zählen vor allem im Winter - da sie auch Austragungsort von FIS Ski-Weltcuprennen sind ${ }^{20}$ aber auch im Sommer zu den erfolgreichsten touristischen Destinationen Südtirols.

\footnotetext{
${ }^{14}$ Ital. Ferrovia del Brennero, die in den Jahren 1864 bis 1867 errichtet wurde und heute Bestandteil der Eisenbahnachse Berlin - Palermo ist; vgl. Laura Facchinelli, Die Eisenbahn Verona Brenner. Geschichte einer bedeutenden Verkehrslinie, Athesia, Bozen, 1995; Günter Denoth, 150 Jahre Eisenbahnen in Tirol, Sutton, Erfurt 2008; Hubert Held, Die Baugeschichte der Brennerbahn 1836-1867: Von München über Alttyrol nach Venedig - aus politischer, ökonomischer und technischer Perspektive, StudienVerlag, Innsbruck-Wien-Bozen, 2018.

${ }^{15}$ Vgl. Evaldo Gaspari, La ferrovia delle Dolomiti. Calalzo - Cortina d'Ampezzo - Dobbiaco. 1921-1964, Bolzano/Bozen, Athesia, 2005; Leonardo Malatesta, Un treno per Cortina, Varese, Pietro Macchione Editore, 2015.

${ }^{16}$ Vgl. https://astat.provinz.bz.it/de (zugegriffen am 30.05.2020).

${ }^{17} \mathrm{Vgl}$. Autonome Provinz Bozen-Südtirol, Landesinstitut für Statistik, Entwicklung im Tourismusjahr, Tourismusjahr 2018/19, astatinfo Nr. 17, 04.2020, S. 6.

${ }^{18}$ Ladin. Gherdëina; ital. Val Gardena.

${ }^{19}$ Ladin. und ital. Alta Badia.

${ }^{20} \mathrm{Vgl}$. https://www.saslong.org/de/news/detail/groeden-und-alta-badia-sind-geruestet-fuer-den-alpinen-skiweltcup-am-letzten-wochenende-vor-weihnachten (zugegriffen am 30.05.2020) und https://www.altabadia.org/de/winter-urlaub-suedtirol/aktivitaeten-und-tipps/fis-ski-world-cup. html (zugegriffen am 30.05.2020).
} 
Dasselbe darf von Cortina d'Ampezzo ${ }^{21}$ gesagt werden: Cortina war bereits in den Jahren 1932 und 1941 Austragungsort der alpinen Ski-Weltmeisterschaften und im Jahre 1956 sogar Austragungsort der VII. Olympischen Winterspiele. 2021 haben wiederum die alpinen Ski-Weltmeisterschaften ${ }^{22}$ stattgefunden und im Jahre 2026 soll Cortina gemeinsam mit Mailand/Milano die XXV. Olympischen Winterspiele $^{23}$ austragen.

Ein guter Teil Südtirols ist bereits im Halbstundentakt mit der Eisenbahn vernetzt, welche inzwischen eine tragende Säule des öffentlichen Personennahverkehrs (ÖPNV) bildet. ${ }^{24}$

Bislang blieben allerdings - zumindest in den letzten 50 Jahren - konkrete Bestrebungen, das Dolomitengebiet mittels Eisenbahn zu erschließen, aus. Ein alpines Eisenbahnangebot - wie wir es aus der Schweiz beispielsweise mit dem genannten Glaciere-Express oder dem Bernina-Express kennen - fehlt bislang gänzlich in den Dolomiten. Während des Ersten Weltkrieges, als das Dolomitengebiet noch Teil der Österreichisch-Ungarische Monarchie war, wurden mehrere ,alpine“ Eisenbahnen betrieben, die teilweise auch in den Kriegsjahren aus militärischen NachschubNotwendigkeiten entstanden sind. ${ }^{25}$ In diesem Zusammenhang sind die ,alte“ Dolomitenbahn (oder Ampezzanerbahn) ${ }^{26}$ von Toblach nach Calalzo, die im Jahre 1964 aufgelassen wurde, sowie die Grödnerbahn ${ }^{27}$ von Klausen nach Wolkenstein/Plan,

\footnotetext{
${ }^{21}$ Ladin. Anpëz oder Anpezo, deutsch (veraltet) Hayden.

${ }^{22} \mathrm{Vgl}$. https://www.cortina2021.com (zugegriffen am 30.05.2020).

${ }^{23} \mathrm{Vgl}$. https://www.milanocortina2026.org (zugegriffen am 30.05.2020).

${ }^{24} \mathrm{Vgl}$. online: https://www.sta.bz.it/de/bahnhoefe-zuege (zugegriffen am 30.05.2020).

${ }^{25}$ Vgl. Walther Schaumann, Die Bahnen zwischen Ortler und Piave in den Kriegsjahren 1915-1918. Einsatz und Leistung der österreichisch-ungarischen und Kaiserlich deutschen Eisenbahnformationen, Bohmann Verlag, Wien-Heidelberg, 1971; Josef Dultinger, Vergessene Vergangenheit. Schmalspurbahnen der k. u. k. Armee zur Dolomitenfront 1915-1918, Verlag Dr. Rudolf Erhard, Rum, 1982. Vgl. auch Piero Muscolino, Ricordi ferrotramviari di viaggi per le Dolomiti con notizie storico-tecniche e immagini sulle ferrovie da tempo soppresse. Ora-Predazzo, Chiusa-Plan, Grafiche Calosci, Cortona, 3. Aufl., 1997, der auf die Bahnlinie Val di Fiemme/Fleimstal - Ora/Auer Cavsalese - Predazzo (S. 133-226) und die Grödnerbahn (S. 227-304) eingeht.

${ }^{26}$ Die „Neue Dolomitenbahn“ darf nicht mit der (,alten“) Dolomitenbahn (it. Ferrovia delle Dolomiti), umgangssprachlich auch „Ampezzaner Bahn“ verwechselt werden. Es handelt sich bei letzterer um eine rund 65 Kilometer lange Schmalspurbahn, die ausgehend von Toblach in Südtirol nach Calalzo in der Provinz Belluno (Region Venetien) führte. Der Bau dieser Bahn begann während des Ersten Weltkrieges, wobei sie von 1921 bis 1964 in Betrieb war; vgl. Evaldo Gaspari, La ferrovia delle Dolomiti. Calalzo - Cortina d'Ampezzo - Dobbiaco. 1921-1964, Athesia, Bolzano/ Bozen 2005; Leonardo Malatesta, Un treno per Cortina, Pietro Macchione Editore, Varese, 2015.

${ }^{27}$ Ital. Ferrovia della Val Gardena, ladin. Ferata de Gherdëina; vgl. Elfriede Perathoner, La ferata de Gherdëina - Die Grödner Bahn, Athesia, Bozen, 2. Aufl., 1997; Elfriede Perathoner, Il trenino della Val Gardena/La ferata de Gherdeina, Bolzano, Athesia, 2017; Vinzenz Peristi, 50 ani da canche i à tëut demetz la ferata de Gherdëina, in: Union di Ladins de Gherdëina (Hrsg.), Calënder de Gherdëina, St. Ulrich in Gröden, 2010, S. 11-40; Elfriede Perathoner/Stefan Planker, Scibla mo 'n iëde. Museum Ladin, San Martin de Tor, 2011; Julius Khu, Der Bau der Grödnerbahn 1915-1916, in: Militärwissenschaftliche Mitteilungen, Nr. 8, August 1937, S. 611-629; Claudio Pedrazzini, La ferrovia della Val Gardena (6 febbraio 1916 - 28 maggio 1960), edizione Associazione Culturale Arnaldo Pocher, Trento, 2011; Angelo Marinoni, Ricordo della ferrovia della Val Gardena, in: Mondo Ferroviario, n. 301, luglio 2012, Editoriale del Garda, Desenzano del Garda, S. 20-29.
} 
die im Jahre 1915 errichtet und im Jahre 1960 eingestellt wurde, hervorzuheben. Beide Bahnen würden heute touristische Hochburgen bedienen.

Eine neue Dolomitenbahn zwischen Bozen und Cortina d'Ampezzo würde heute neun Gemeinden mit rund 30.000 Einwohnern, ${ }^{28}$ rund 55.000 Gästebetten ${ }^{29}$ und weitere rund 30.000 Betten in Zweitwohnungen, sowie jährlich 1,3 Millionen Ankünfte und 6,8 Millionen Nächtigungen ${ }^{30}$ bedienen.

Aus ethnopolitischer Sicht würde die neue Dolomitenbahn das dolomitenladinische Gebiet, d. h. das geschlossene Siedlungsgebiet der dolomitenladinischen Bevölkerung, ${ }^{31}$ das im Jahre $1923^{32}$ und $1927^{33}$ auf drei verschiede Provinzen aufgeteilt wurde, zumindest zwischen Gröden und Ampezzo eisenbahntechnisch verbinden.

\subsection{Die Verpflichtung zum nachhaltigen Landschafts- und Umweltschutz in den Alpen und Dolomiten}

Am 26. Juni 2009 hat das Welterbe-Komitee der UNESCO ${ }^{34}$ im Rahmen seiner 33. Sitzung in der Stadt Sevilla in Spanien weite Teile der Dolomiten in die Liste des Welterbes der Menschheit aufgenommen.

\footnotetext{
${ }^{28}$ Autonome Provinz Bozen-Südtirol, Landesinstitut für Statistik, Südtirol in Zahlen 2019, Stand 31.12.2018.

${ }^{29}$ Autonome Provinz Bozen-Südtirol, Landesinstitut für Statistik, Beherbergungsbetriebe und Betten nach Kategorie, in: https://qlikview.services.siag.it/QvAJAXZfc/opendoc_notool.htm?document=tourismus.qvw\&host=QVS\%40titan-a\&anonymous=true (zugegriffen am 30.05.2020).

${ }^{30}$ IDM Südtirol, Statistiken, online: https://www.idm-suedtirol.com/de/tourismus/statistiken.html (30.05.2020).

${ }^{31}$ Vgl. Christoph Perathoner, Die Dolomitenladiner 1848-1918. Ethnisches Bewusstsein und politische Partizipation, Folio Verlag Wien, Bozen, 1998, S. 23. „Mit dem Terminus Dolomitenladiner bezeichnet man die ladinische Bevölkerung in den vier Talschaften um das Sellamassiv. Es sind: das Gadertal mit dem Seitental Enneberg, das Grödnertal, das Fassatal, Buchenstein mit Colle Santa Lucia und Cortina d'Ampezzo“. Allgemein siehe: Peter Hilpold/Christoph Perathoner (Hrsg.), Die Ladiner. Eine Minderheit in der Minderheit, Neuer Wissenschaftlicher Verlag, Wien, 2005.

${ }^{32}$ Vgl. Königliches Dekret, 21. Januar 1923, Nr. 93, in: Gazz. Uff., 30. Januar 1923, Nr. 24; Vgl auch Christoph Perathoner, Der Schutz der ladinischen Minderheit in Südtirol, in: Peter Hilpold/ Christoph Perathoner (Hrsg.), Die Ladiner. Eine Minderheit in der Minderheit, Neuer Wissenschaftlicher Verlag, Wien, 2005, S. 50 u. 54.

${ }^{33}$ Vgl. Königliches Dekret, 2. Januar 1927, Nr. 1, in: Gazz. Uff., 11. Januar 1927, Nr. 7 (Schaffung der Provinz Bozen).

${ }^{34}$ UNESCO = engl. United Nations Educational, Scientific and Cultural Organization; deutsch Organisation der Vereinten Nationen für Erziehung, Wissenschaft und Kultur, in: https://en.unesco. org (zugegriffen am 30.05.2020).
} 
Die landschaftliche Schönheit dieses Gebietes sowie ihre geologische und geomorphologische Bedeutung wurden als weltweit einzigartig eingestuft. ${ }^{35}$ Daraus entsteht für die öffentlichen Körperschaften eine klare Verpflichtung zum nachhaltigen Schutz der Landschaft und Umwelt.

Der ehemalige Landeshauptmann-Stellvertreter Südtirols und Präsident der Stiftung Dolomiti - Dolomiten - Dolomites - Dolomitis UNESCO ${ }^{36}$ Richard Theiner machte im Rahmen der Aktion des Landes Südtirols Dolomitesvives ${ }^{37}$ darauf aufmerksam, dass jährlich rund 1,2 Millionen Fahrzeuge allein rund um den SellaStock in den Dolomiten unterwegs sind, was einer Anzahl von 2300 Fahrzeuge pro Tag entspricht. ${ }^{38}$

Es gibt mittlerweile auch Beispiele von Tourismusdestinationen, die sich autofrei entwickeln wollen und dabei auf die Erreichbarkeit mittels Bahn setzen, so z. B. Zermatt im schweizer Kanton Wallis. ${ }^{39}$ Die Dolomitenbahn könnte ein geeignetes Instrument für ähnliche Entwicklungen in den Dolomiten darstellen.

\subsection{Die Trasse}

Die Bahntrasse der neuen Dolomitenbahn würde in Bozen starten, da mit der Fertigstellung des BBT und der Dynamisierung der TEN-Linie Berlin-Palermo viele Züge in Südtirol - um eine schnelle Anbindung der europäischen Millionenstädte entlang der TEN-Linie zu garantieren - wahrscheinlich nur mehr dort - wenn überhaupt - halten werden. Da die neue Dolomitenbahn vor allem auch touristischen Interessen zu Grunde liegt und Touristen aus der ganzen Welt ansprechen soll, scheint es unumgänglich, diese beim Südtiroler Hauptbahnhof in Bozen abzuholen. ${ }^{40}$

Die Eisenbahntrasse soll von Bozen über das Schlern-Hochplateau (Völs, Seis, Kastelruth) durch das Grödental (Pontives, St. Ulrich, St. Christina, Wolkenstein)

\footnotetext{
${ }^{35} \mathrm{Vgl}$. https://naturparks.provinz.bz.it/dolomiten-unseco-welterbe.asp (zugegriffen am 30.05.2020). ${ }^{36}$ Vgl. https://www.dolomitiunesco.info (30.05.2020). Die Stiftung Dolomiti - Dolomiten - Dolomites - Dolomitis UNESCO wurde 2010 von fünf Provinzen (Belluno, Bozen, Pordenone, Trient, Udine) und zwei Regionen (Friaul-Julisch Venetien und Veneto) gegründet, um eine effiziente und koordinierte Verwaltung des Dolomiten-Welterbes zu gewährleisten.

${ }^{37} \mathrm{Vgl}$. http://www.provinz.bz.it/news/de/news.asp?art=615194 (zugegriffen am 30.05.2020). Die Aktion Dolomitesvives, ladin. ,für lebendige Dolomiten“, wurde am 15. Mai 2017 in Bozen vorgestellt und zielt auf eine Verkehrsreduzierung in den Dolomiten ab, so soll beispielsweise das Sella-Joch neun Mal im Juli und August für Fahrzeuge mit Verbrennungsmotor von 9 bis 16 Uhr gesperrt werden.

${ }^{38} \mathrm{Vgl}$. http://www.provinz.bz.it/tourismus-mobilitaet/mobilitaet/aktuelles.asp?news_action=4\&news_article_id=588349 (zugegriffen am 30.05.2020).

${ }^{39} \mathrm{Vgl}$. https://www.zermatt.ch/anreise (zugegriffen am 30.05.2020).

${ }^{40}$ Es gibt Projekte der STA AG, die bestrebt sind, die alte Grödnerbahn wieder aufleben zu lassen. Diese Projekte würden diese Bahn in Klausen oder in Waidbruck starten lassen und bis Wolkenstein/Plan führen. Entsprechende Studien wurden von den Gemeindeverwaltungen Grödens im Laufe des letzten Jahrzehnts mitfinanziert.
} 
ins Gadertal (Kolfuschg, Corvara, Stern, St. Kassian) und schließlich über den Valparola- und Falzaregopass bis nach Cortina d'Ampezzo führen. Die Gesamtstrecke wäre ca. $85 \mathrm{~km}$ lang und in 2 Stunden und 17 Minuten zu bewältigen.

Das Projekt sieht 19 Bahnhöfe/Haltestellen vor, wobei die Züge auf der Gesamtstrecke im 30-Minuten-Takt und in Gröden im 15-Minuten-Takt verkehren würden. Als Haltestellen wurden strategische Knotenpunkte gewählt, um sich auch der bereits bestehenden Aufstiegsanlagen zu bedienen, die Teil der wichtigen touristischen Infrastrukturen sind. Die Bahn könnte auch nachts oder in den Wintermonaten für den Güterverkehr genutzt werden.

Nachstehend findet sich eine Übersicht der Bahnhöfe mit den zu bewältigenden Kilometern ab dem Startbahnhof in Bozen samt der jeweils notwendigen Zeit im simulierten Normalbetrieb: ${ }^{41}$

$\begin{array}{lll}\text { Nr. 1: Bozen } & \mathrm{km} 0,0 & 0 \text { min } \\ \text { Nr. 2: Blumau } & \mathrm{km} \mathrm{8,3} & 8 \mathrm{~min} \\ \text { Nr. 3: Völs } & \mathrm{km} \mathrm{14,9} & 22 \mathrm{~min} \\ \text { Nr. 4: Seis } & \mathrm{km} \mathrm{20,9} & 30 \mathrm{~min} \\ \text { Nr. 5: Kastelruth } & \mathrm{km} \mathrm{24,5} & 34 \mathrm{~min} \\ \text { Nr. 6: Pontives } & \mathrm{km} \mathrm{30,5} & 43 \mathrm{~min} \\ \text { Nr. 7: St. Ulrich } & \mathrm{km} \mathrm{33,7} & 46 \mathrm{~min} \\ \text { Nr. 8: St. Christina } & \mathrm{km} \mathrm{38,7} & 51 \mathrm{~min} \\ \text { Nr. 9: Wolkenstein } & \mathrm{km} \mathrm{41,2} & 59 \mathrm{~min} \\ \text { Nr. 10: Plan de Gralba } & \mathrm{km} \mathrm{43,4} & 63 \mathrm{~min} \\ \text { Nr. 11: Grödnerjoch } & \mathrm{km} \mathrm{46,9} & 70 \mathrm{~min} \\ \text { Nr. 12: Kolfusch } & \mathrm{km} \mathrm{53,1} & 86 \mathrm{~min} \\ \text { Nr. 13: Corvara } & \mathrm{km} \mathrm{54,1} & 89 \mathrm{~min} \\ \text { Nr. 14: Stern } & \mathrm{km} \mathrm{58,1} & 92 \mathrm{~min} \\ \text { Nr. 15: St. Kassian } & \mathrm{km} \mathrm{61,0} & 95 \mathrm{~min} \\ \text { Nr. 16: Falzarego-Pass } & \mathrm{km} \mathrm{70,3} & 110 \mathrm{~min} \\ \text { Nr. 17: Cinque Torri } & \mathrm{km} \mathrm{72,0} & 117 \mathrm{~min} \\ \text { Nr. 18: Pocol } & \mathrm{km} \mathrm{77,0} & 125 \mathrm{~min} \\ \text { Nr. 19: Cortina d'Ampezzo } & \mathrm{km} \mathrm{83,0} & 137 \mathrm{~min}\end{array}$

\subsection{Das Rollmaterial - Eine Zahnradbahn die gleichzeitig normale Eisenbahn ist.}

Für die neue Dolomitenbahn ist ein eigenes Rollmaterial notwendig, um den unterschiedlichen Notwendigkeiten des Geländes gerecht zu werden.

So wird ein gemischtes Bahnsystem aus Adhäsions- und Zahnradantrieb vorgeschlagen, welches steile Gelände als Zahnradbahn meistern und flachere Strecken als Adhäsionsbahn bewältigen kann.

\footnotetext{
${ }^{41}$ Ein Video der des Verlaufes der „Neuen Dolomitenbahn“ findet sich unter https://www.sad.it/ sites/default/files/02.mp4 (zugegriffen am 30.05.2020) wie auch unter https://www.tageszeitung. it/2016/07/30/die-spektakulaere-bahn/ (zugegriffen am 30.05.2020).
} 
Die Vorteile eines solchen Systems sind nicht unerheblich: Die Länge der Bahnlinie wird verringert, große Eingriffe - lange Tunnels oder Brücken - werden vermieden und für die Fahrgäste würde die Fahrt ein Naturerlebnis in den Dolomiten darstellen. Während die Adhäsionsstrecke $35 \mathrm{~km}$ betragen soll, sind für die Zahnradstrecke $40 \mathrm{~km}$ vorgesehen, wobei die maximale Steigung bei $12 \%$ liegt.

\subsection{Die Kosten und die Finanzierung}

Das Projekt Dolomitenbahn wurde im Jahr 2016 mit Kosten von ca. 1,8 Milliarden Euro veranschlagt: Davon würden 1,6 Milliarden Euro für den Bau der Strecke und 200 Millionen Euro für den Kauf der Züge eingeplant.

Als Finanzierungsmodell schlug die SAD Nahverkehr AG eine öffentlichprivate Partnerschaft (ÖPP) ${ }^{42}$ vor, bei welcher im Kernpunkt dem privaten Unternehmen als Gegenleistung für den Bau der Bahnlinie eine möglichst lange öffentlich-rechtliche Konzession für den Betrieb der Bahnlinie eingeräumt werden sollte.

Darüber hinaus sollte bei einem Projekt dieser Größenordnung aber auch eine direkte Beteiligung der Länder/Regionen, des Staates und der EU erfolgen. Im Modell des vorgenannten privaten Unternehmenssollte diese zusätzliche Finanzierung zu 50 \% durch öffentliche Zuschüsse (davon 30-40 \% durch die Europäische Union und die restlichen 10-20\% durch das Land Südtirol und die Region Veneto), zu $35 \%$ durch Darlehen (z. B. durch die Europäische Investitionsbank oder die Cassa Depositi e Prestiti) und zu $15 \%$ durch Risikokapital, also durch von privaten oder institutionellen Anlegern bereitgestelltes Eigenkapital (equity) erfolgen.

Bei einer angenommenen Konzessionsdauer von 50 Jahren würde dieses Modell einen jährlichen Betriebszuschuss von rund 50 Million Euro bedürfen.

Die Neue Dolomitenbahn würde neun Gemeinden mit zwar nur rund 30.000 Einwohnern, jedoch mit einem starken, durch den Tourismus generiertes Bruttoinlandsprodukt, bedienen. So könnte der veranschlagte Kosten-Betrag von 1,8 Milliarden Euro zu einem beträchtlichen Teil durch Einsparungen sowie durch zusätzliche Steuereinnahmen, welche durch die Bahn generiert würden, gedeckt werden. Im simulierten Modell würden beispielsweise Einsparung aus reduzierten Busdiensten (ca. 3 Millionen Euro), der Besteuerung des Einkommens der Betreibergesellschaft (ca. 10 Millionen Euro), den Steuern aus zusätzlichem Bruttoinlandsprodukt bei einer zusätzlichen Million an Nächtigungen (ca. 32 Millionen Euro) oder eventuelle zusätzliche Tourismusabgaben (ca. 16 Millionen Euro) eine zusätzliche Finanzierungshilfe generiert. Die Ersparnisse bzw. Steuereinnahmen der eben genannten ersten drei Positionen, welche einen Gesamtbetrag von 45 Millionen Euro ausmachen, würden ausschließlich durch die Existenz der Bahn generiert. Des Weiteren würde sich im genannten Modell bei vorgesehenen jährlichen Betriebskosten

\footnotetext{
${ }^{42}$ Engl. $P P P=$ public-private-Partnership.
} 
von 16 Millionen Euro und Einnahmen von 21 Millionen Euro sogar ein Verwaltungsüberschuss in Höhe von 5 Millionen Euro ergeben.

\section{Abschließende Betrachtungen}

Die Idee einer alpinen Eisenbahnlinie von Genf nach Venedig als verkehrstechnische Ergänzung zu den alpenquerenden Nord-Südkorridoren der TEN-V-Politik der Europäischen Union verdient vertieft zu werden. Dies allein deshalb, weil eine derart gestaltete Eisenbahntrasse neben dem sanften Mobilitäts- und Transportaspekt, sowohl im schweizerischen wie im italienischen Teil, einen touristischen und insgesamt wirtschaftlichen Mehrwert generieren könnte.

Dieser Umstand spiegelt sich in besonderer Weise gerade bei der „Neuen Dolomitenbahn" wider. Zum einen ist sie so konzipiert - und das wurde oben beim simulierten Zeitplan im Normalbetrieb dargestellt -, dass sie die Funktion des öffentlichen Personennahverkehrs übernehmen und verbessern kann. Zum anderen wäre sie eine touristische Attraktion - vor allem für das dolomitenladinische Gebiet (Gröden-Gadertal-Cortina) - und würde eine umweltbewusste und ökologisch vertretbare Erschließungsmöglichkeit für das einzigartige UNESCO Weltnaturerbe der Dolomiten darstellen.

Die Trassierung der Bahnlinie der Neuen Dolomitenbahn würde über eine Strecke von rund $85 \mathrm{~km}$ zwischen Bozen und Cortina d'Ampezzo über das Schlerngebiet, das Grödental, das Hochabteital und den Falzarego Pass verlaufen und dabei neun Gemeinden mit rund 30.000 Anrainern durchqueren, die in zwei unterschiedlichen italienischen Regionen (und Provinzen) liegen. Die Neue Dolomitenbahn würde drei Sprachgebiete - und zwar das italienische, das deutsche und das ladinische - durchqueren.

Die Fahrzeit würde insgesamt zwischen 120 und 150 Minuten betragen, wenn sie rund 20 Haltestellen bzw. Bahnhöfe anfahren würde. Das Fahrdienstangebot könnte im 30-Minuten-Takt verlaufen, für das touristische Gröden sogar im 15-Minuten-Takt.

Die Gesamtinvestition dürfte grob geschätzt bei 1,8 Milliarden Euro liegen, wobei rund 1,6 Milliarden Euro für den Bau und der Rest für den Erwerb von Zügen, die sowohl mit einem Adhäsions- wie auch mit einem Zahnrad-System ausgestattet wären, eingesetzt werden müssten. Dieser technologische Ansatz, nämlich die Alternanz von Adhäsionstechnologie bei der Fortbewegung in ebenem Gelände und das Zahnradsystem bei den Steigungen (die maximale Steigung beträgt beim vorliegenden Projekt ca. $12 \%$ ) ist innovativ und in der Praxis heute noch selten.

Grundsätzlich ist die Idee, dieses Projekt über eine öffentlich-private Partnerschaft (ÖPP) zu verwirklichen, sicherlich zu befürworten. Aufgrund der Größe des Projektes ist eine direkte finanzielle Beteiligung der Länder/Regionen, des Staates und der EU unumgänglich. Eine private Beteiligung würde zudem die unternehmerischen Elemente einfließen lassen, die bei Pionierleistungen einen klaren Mehrwert bringen können. Zudem könnte eine langjährige Konzession an ein 
privates Unternehmen, das an der Planung und Verwirklichung der Bahn beteiligt ist, die Anfangsschwierigkeiten besser bewältigen und eine gewisse Kontinuität und Beständigkeit des Projektes in den ersten Jahrzehnten nach dem Bau der Linie garantieren.

Die Grundvoraussetzung für die Verwirklichung ist zum einen die Akzeptanz der Anrainerbevölkerung, zum anderen aber auch ein politischer Wille, der nicht nur auf regionaler sondern auch auf staatlicher Ebene vorhanden sein muss, zumal ohne den Einsatz Italiens ein Zugriff zu den Fördermöglichkeiten der Europäischen Union kaum möglich sein dürfte.

Open Access Dieses Kapitel wird unter der Creative Commons Namensnennung 4.0 International Lizenz (http://creativecommons.org/licenses/by/4.0/deed.de) veröffentlicht, welche die Nutzung, Vervielfältigung, Bearbeitung, Verbreitung und Wiedergabe in jeglichem Medium und Format erlaubt, sofern Sie den/die ursprünglichen Autor(en) und die Quelle ordnungsgemäß nennen, einen Link zur Creative Commons Lizenz beifügen und angeben, ob Änderungen vorgenommen wurden.

Die in diesem Kapitel enthaltenen Bilder und sonstiges Drittmaterial unterliegen ebenfalls der genannten Creative Commons Lizenz, sofern sich aus der Abbildungslegende nichts anderes ergibt. Sofern das betreffende Material nicht unter der genannten Creative Commons Lizenz steht und die betreffende Handlung nicht nach gesetzlichen Vorschriften erlaubt ist, ist für die oben aufgeführten Weiterverwendungen des Materials die Einwilligung des jeweiligen Rechteinhabers einzuholen. 